\title{
Literature Review and Previous Flood Barrier Tests - Slide Package
}

Sai Zhang, Zhegang Ma

March 2020

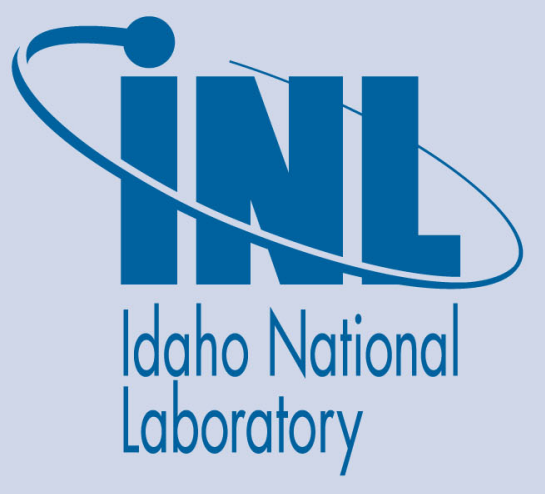

The INL is a U.S. Department of Energy National Laboratory operated by Battelle Energy Alliance 


\section{Literature Review and Previous Flood Barrier Tests - Slide Package}

Sai Zhang, Zhegang Ma

March 2020

Idaho National Laboratory Idaho Falls, Idaho 83415

http://www.inl.gov

Prepared for the U.S. Department of Energy Office of Nuclear Energy Under DOE Idaho Operations Office Contract DE-AC07-05ID14517 


\section{Literature Review and Previous Flood Barrier Tests}

Sai Zhang, Ph.D.

Zhegang Ma, Ph.D., P.E.

Idaho National Laboratory

NRC Flood Barrier Testing Strategies Workshop

March 12, 2020

Rockville, MD USA 


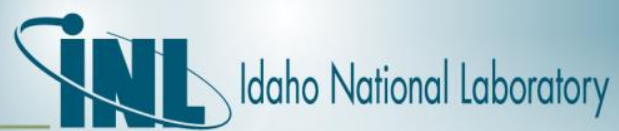

\section{Presentation Outline}

$>$ Literature Review

$>$ Plant-Specific Flood Barrier Types and Performances

$>$ Examples of Previous Flood Barrier Tests 


\section{Literature Review}

$>$ Reviewed Materials from a Variety of Sources

$\square$ Domestic Agencies

- United States Nuclear Regulatory Commission (NRC)

- United States Army Corps of Engineers (ACE)

$\square$ International Agency

- Organisation for Economic Co-operation and Development Nuclear Energy Agency (OECD NEA)

$\square$ Industry and Academia

- Nuclear Energy Institute (NEI)

- Electric Power Research Institute (EPRI)

- Licensee flooding walkdown reports

- Nuclear power plant (NPP) decommissioning information

- Idaho National Laboratory (INL) and Idaho State University (ISU)

- Relevant publications in scientific journals and conferences

- Publicly available information from flood barrier vendors 


\section{Literature Review (cont.)}

$>$ List of Reviewed Materials

$\square$ NRC

- Materials Related to Flood Barriers

- Regulatory Guide 1.102, Rev. 1, "Flood Protection for Nuclear Power Plants," 1976

- Japan Lessons-learned Project Directorate, Interim Staff Guidance, JLDISG-2012-05, Rev.0, "Guidance for Performing the Integrated Assessment for External Flooding," 2012

- Draft NUREG report, "Development of a Performance Testing Protocol for Nuclear Power Plant Flood Penetration Seals," in progress

- Reports prepared by NRC contractors, including Fire Risk Management, Inc. and Center for Nuclear Waste Regulatory Analyses

- Materials Related to Fire Barriers or Fire Tests

- NUREG/CR-0152, "Development and Verification of Fire Tests for Cable Systems and System Components," 1978

- NUREG/CR-2377, "Tests and Criteria for Fire Protection of Cable Penetrations," 1981

- NUREG-1552, "Fire Barrier Penetration Seals in Nuclear Power Plants," 1996 


\section{Literature Review (cont.)}

$>$ List of Reviewed Materials (cont.)

$\square$ ACE Engineering Research and Development Center (ERDC)

- ERDC TR-07-3, "Flood-Fighting Structures Demonstration and Evaluation Program: Laboratory and Field Testing in Vicksburg, Mississippi," 2007

- ERDC/CHL TR-15-3, "Technical Basis for Flood Protection at Nuclear Power Plants," 2015

$\square$ OECD NEA

- NEA draft report, "Concepts and Terminology for Protecting Nuclear Installations from Flood Hazards," in progress

$\square$ NEI

- NEI 12-07, Rev. 0-A, "Guidelines for Performing Verification Walkdowns of Plant Flood Protection Features," 2012

$\square$ EPRI

- Product 3002005423, "Flood Protection Systems Guide," 2015

- Presentation, "External Flood Seal Risk-Ranking Process," 2019 


\section{Literature Review (cont.)}

$>$ List of Reviewed Materials (cont.)

$\square$ Licensee Walkdown Reports

- Flooding walkdown reports of a reference NPP, 2013-2014

$\square$ INL \& ISU

- Pope et al., "Light Water Reactor Sustainability Program, Nuclear Power Plant Mechanical Component Flooding Fragility Experiments Status (INL/EXT-1742728)," 2017

- Wells et al., "Non-watertight door performance experiments and analysis under flooding scenarios," Results in Engineering, 2019

$\square$ Others (ongoing)

- NPP decommissioning info, vendor info, and scientific publications

\section{$>$ Outputs of Literature Review}

$\square$ Generic categorization of flood barriers in NPPs

$\square$ Plant-specific flood barrier types and performances (to be presented in part II)

$\square$ Existing and potential flood barrier testing facilities

$\square$ Examples of previous flood barrier tests (to be presented in part III)

$\square$ Insights for future flood barrier testing strategy development 


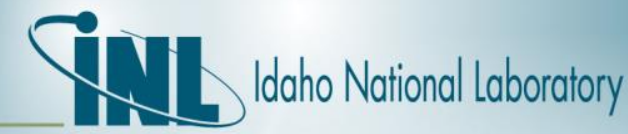

\section{Plant-Specific Flood Barrier Types and Performances}

$>$ Reviewed Flooding Walkdown Reports of a Reference Plant

$\square$ Most of inspected protection features in the plant are flood barriers

$\square$ Most of inspected flood barriers are incorporated into the plant

\begin{tabular}{|l|l|l|l|}
\hline Feature Type & Classified as Barrier & Barrier Type & Percentage \\
\hline Seal & Yes & Incorporated & $79.11 \%$ \\
\hline Structure & Yes & Incorporated & $7.75 \%$ \\
\hline Drain & No & n/a & $7.51 \%$ \\
\hline Scupper & No & n/a & $3.76 \%$ \\
\hline Dike & Yes & Exterior & $0.94 \%$ \\
\hline Sump & No & n/a & $0.70 \%$ \\
\hline Monitor Well & No & n/a & $0.23 \%$ \\
\hline Percentage of Barrier-Type Features & & $87.79 \%$ \\
\hline Percentage of Non-Barrier-Type Features & $12.21 \%$ \\
\hline
\end{tabular}




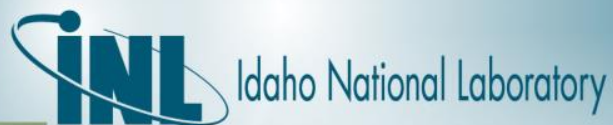

II. Plant-Specific Flood Barrier Types and Performances (cont.)

$>$ Flood Barrier Types in the Reference Plant

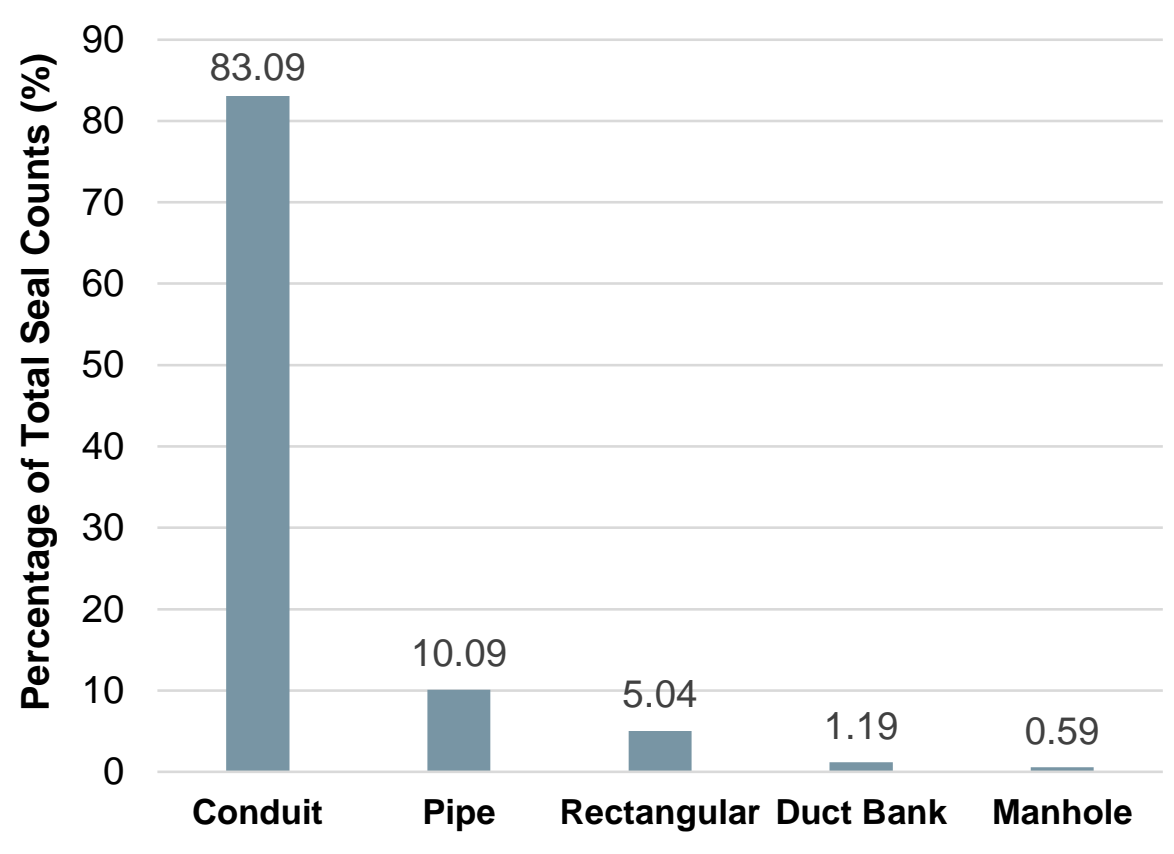

Penetration Seals

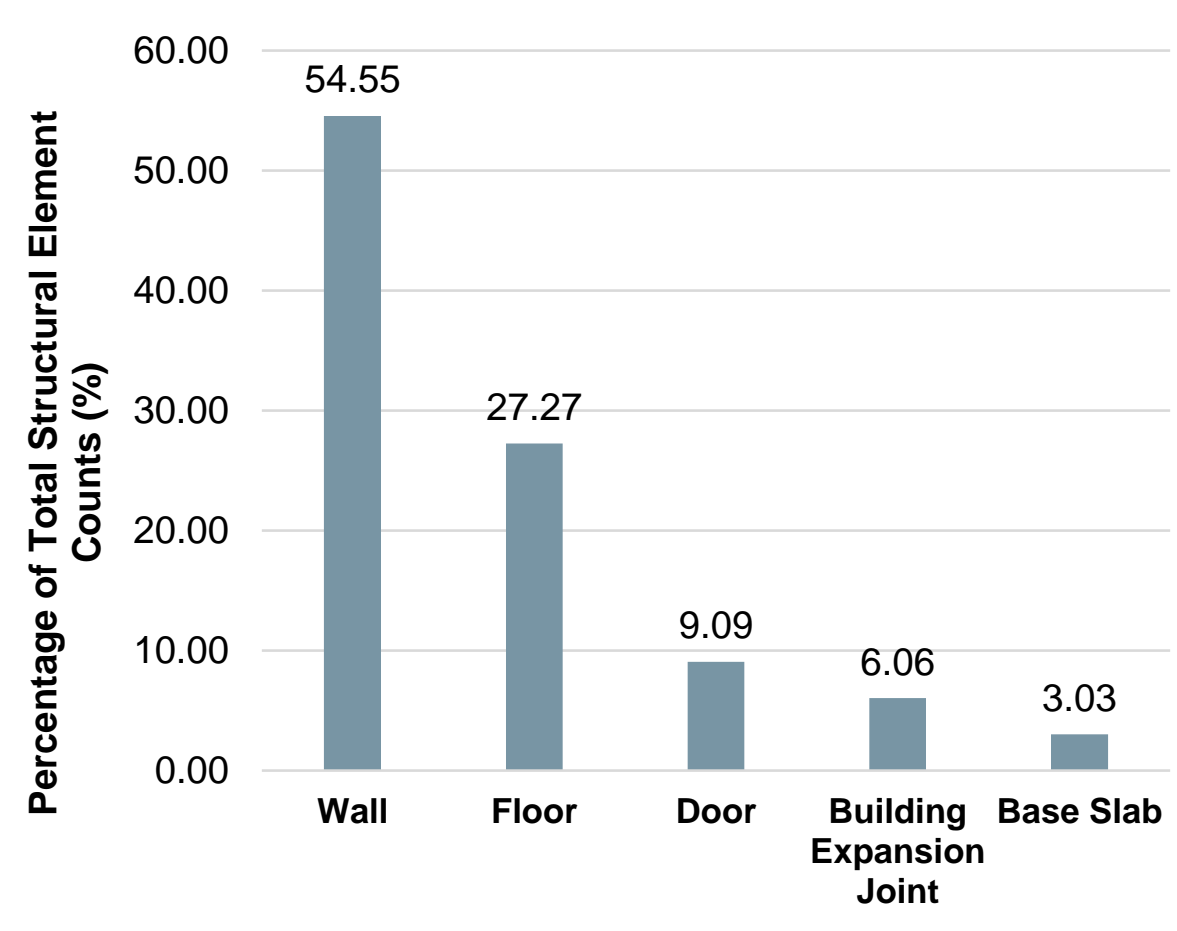

Structural Elements 
II. Plant-Specific Flood Barrier Types and Performances (cont.)

\section{$>$ Flood Barrier Performances in the Reference Plant}

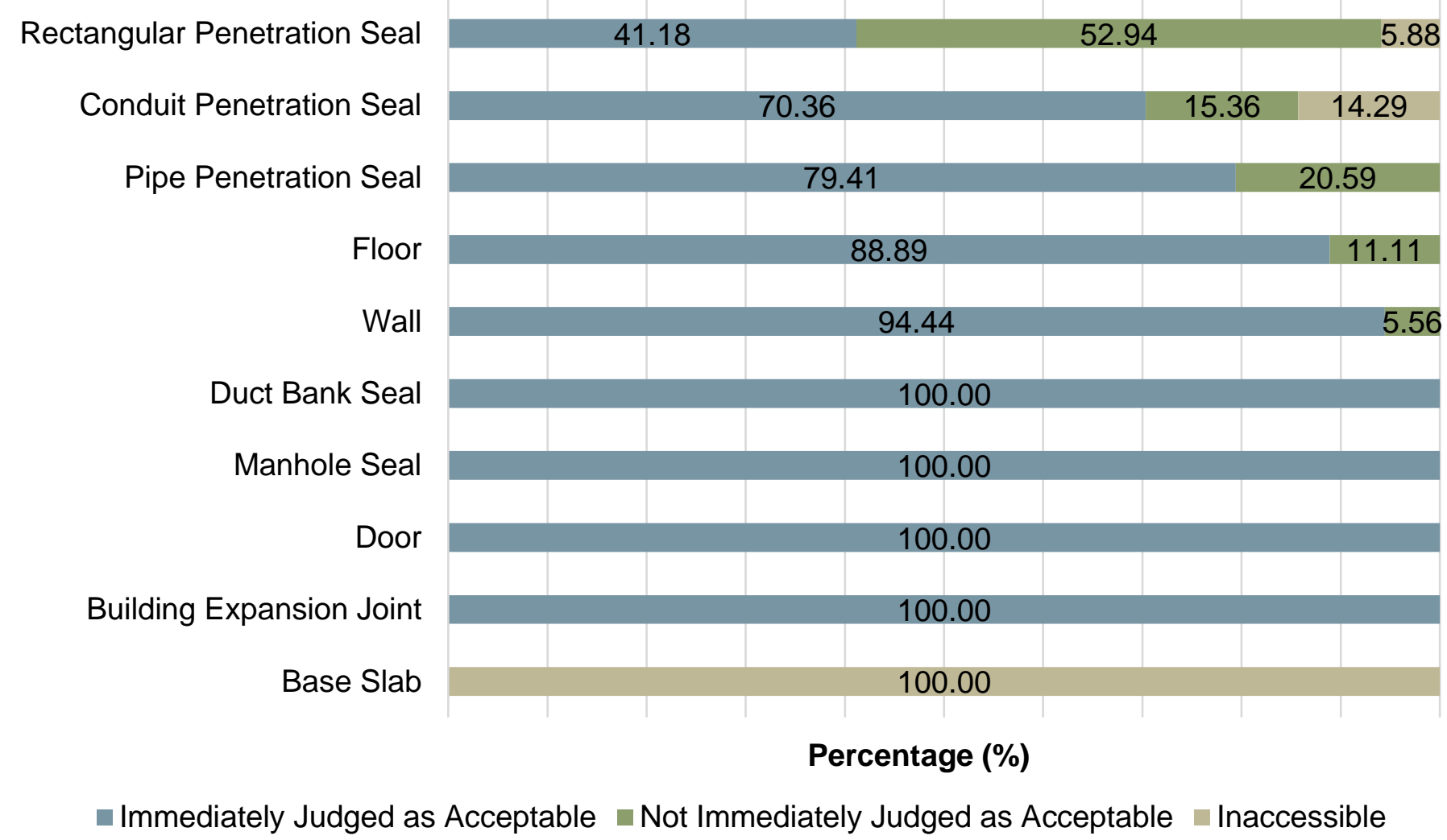




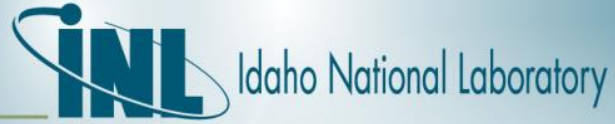

II. Plant-Specific Flood Barrier Types and Performances (cont.)

$>$ Flood Barrier Performances in the Reference Plant (cont.)

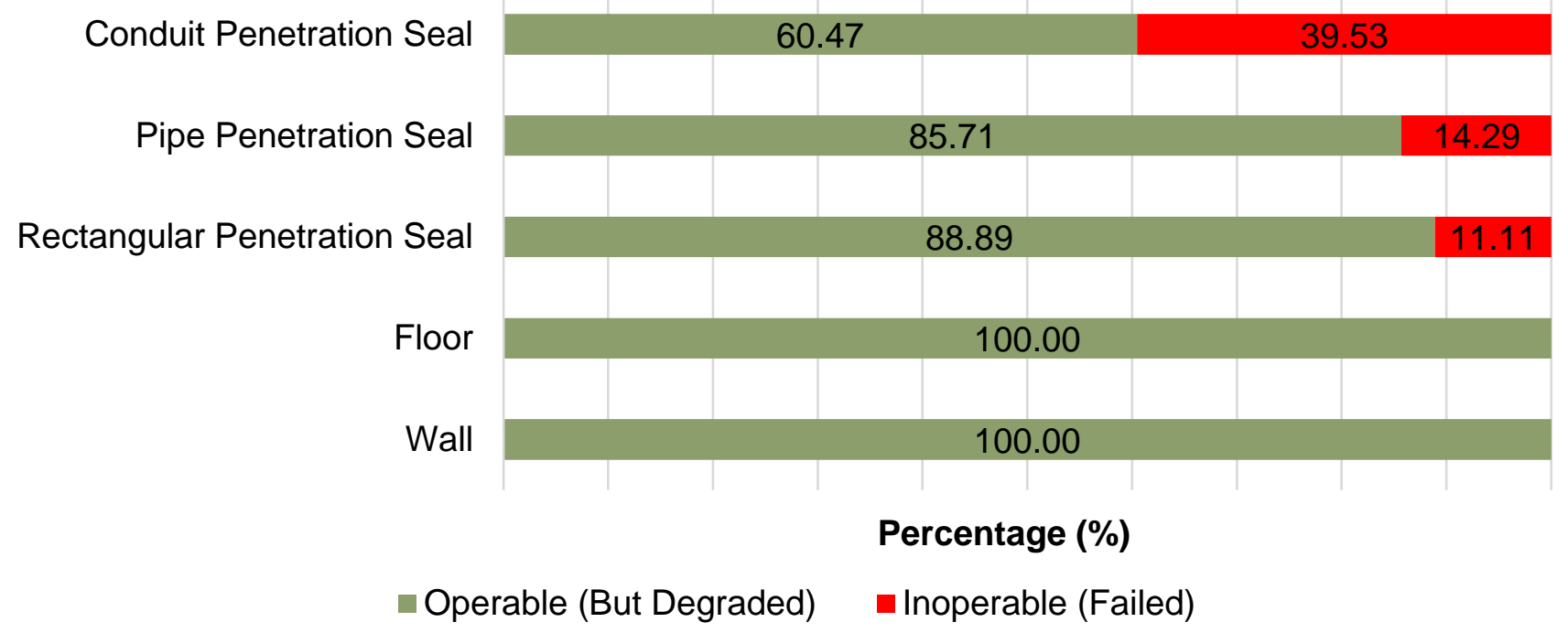


II. Plant-Specific Flood Barrier Types and Performances (cont.)

\section{$>$ Degraded Flood Barriers in the Reference Plant}

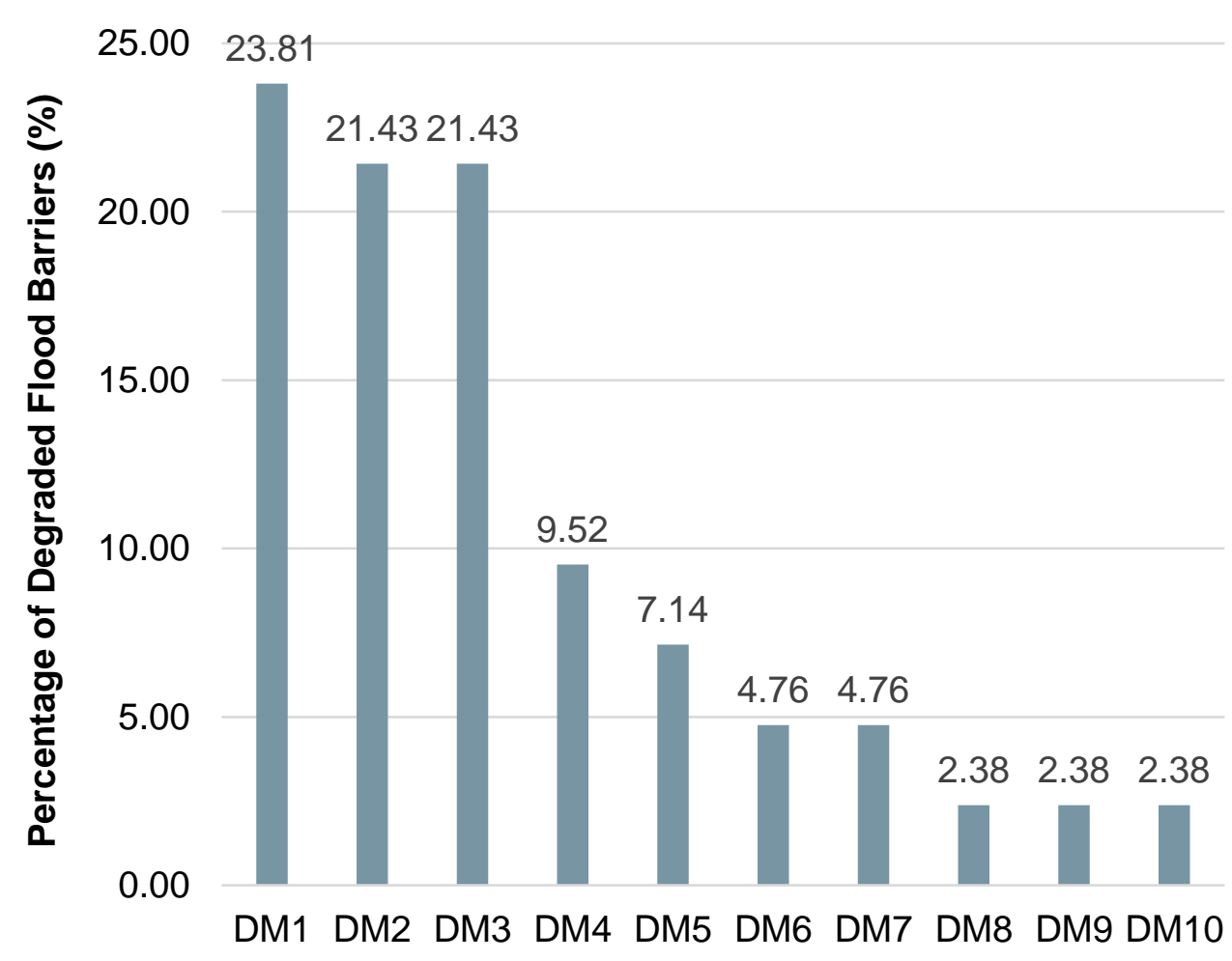

\begin{tabular}{|c|c|}
\hline No. & Degradation Mode (DM) \\
\hline DM1 & $\begin{array}{l}\text { Corrosion on penetration and signs of water } \\
\text { seepage on wall. }\end{array}$ \\
\hline DM2 & $\begin{array}{l}\text { Staining on wall below penetration or at } \\
\text { construction joints of penetration and } \\
\text { immediately below. }\end{array}$ \\
\hline DM3 & No seal could be observed for this penetration. \\
\hline DM4 & Staining on wall and corrosion on penetration. \\
\hline DM5 & $\begin{array}{l}\text { Extensive corrosion on penetration sleeves and } \\
\text { stalactite growth underneath the penetration and } \\
\text { cap. }\end{array}$ \\
\hline DM6 & $\begin{array}{l}\text { Cracks greater than } 0.04 \text { " wide in the wall/floor } \\
\text { slab. }\end{array}$ \\
\hline DM7 & $\begin{array}{l}\text { Penetration covered by a catch and inaccessible. } \\
\text { Staining on the wall below the catch. }\end{array}$ \\
\hline DM8 & $\begin{array}{l}\text { Staining on penetration and signs of water } \\
\text { seepage on wall. }\end{array}$ \\
\hline DM9 & $\begin{array}{l}\text { Cracks greater than } 0.04 \text { " wide in the grout } \\
\text { sealing penetration and slight staining below } \\
\text { pipes. }\end{array}$ \\
\hline DM10 & $\begin{array}{l}\text { Due to an obstructed view, an internal seal for } \\
\text { this pipe sleeve could not be verified. }\end{array}$ \\
\hline
\end{tabular}


II. Plant-Specific Flood Barrier Types and Performances (cont.)

\section{$>$ Failed Flood Barriers in the Reference Plant}

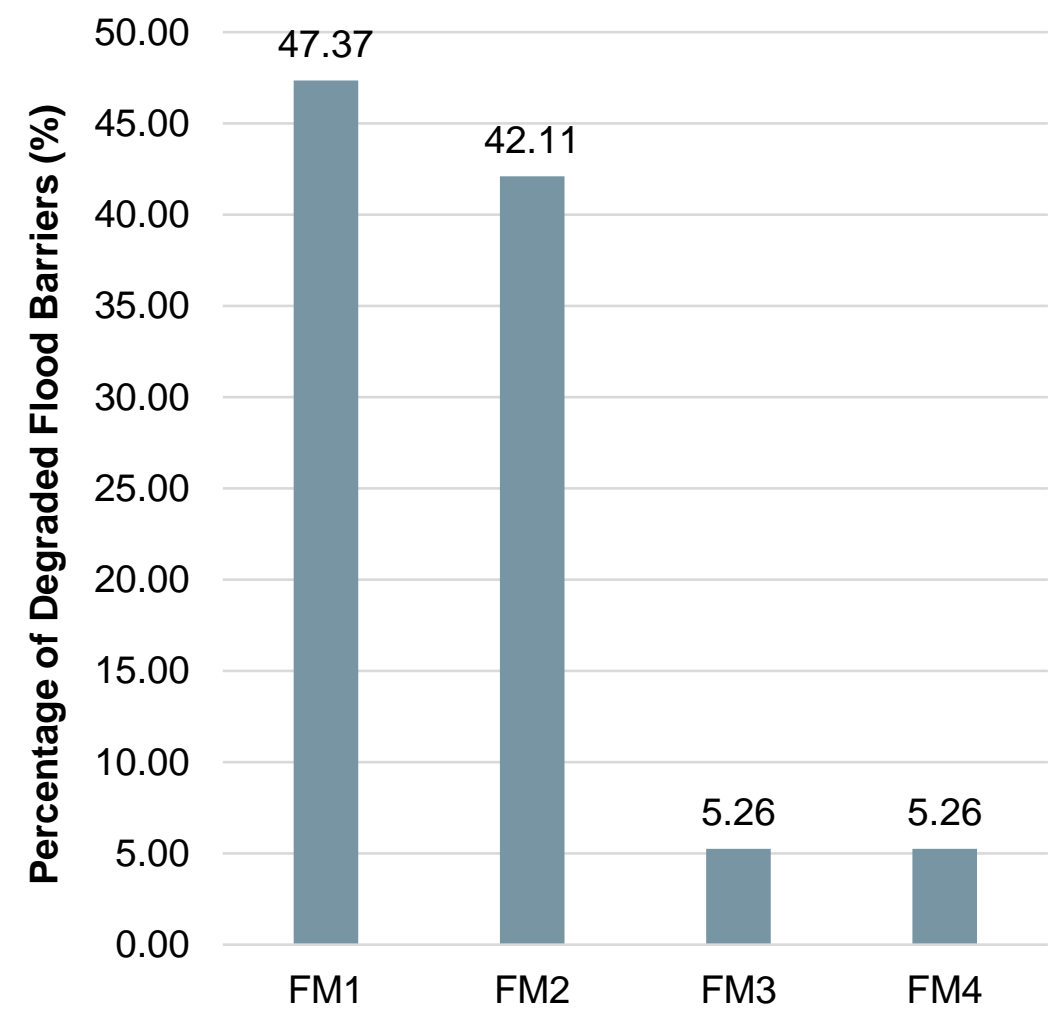

\begin{tabular}{|l|l|}
\hline No. & Failure Mode (FM) \\
\hline FM1 & $\begin{array}{l}\text { Penetration seals appeared severely degraded. } \\
\text { Signs of past water intrusion on walls underneath. }\end{array}$ \\
\hline FM2 & $\begin{array}{l}\text { Water intrusion through penetrations observed at } \\
\text { roughly } 40 \text { drops per minute during a light } \\
\text { rainstorm. }\end{array}$ \\
\hline FM3 & $\begin{array}{l}\text { Penetrating conduit was cut and uncapped. } \\
\text { FM4 }\end{array}$ \\
\hline \\
Penetrating conduit was cut and uncapped. A seal \\
inside the penetrating conduit was not visible.
\end{tabular}


II. Plant-Specific Flood Barrier Types and Performances (cont.)

> Performance Metrics of Flood Barriers in the Reference Plant

$\square$ Could possibly act as an input for development of flood barrier testing strategy

- Failure probability could be one of multiple factors to be considered for test prioritization

\begin{tabular}{|l|l|l|l|}
\hline \multirow{2}{*}{ Flood Barrier } & Probability & \multicolumn{2}{l|}{} \\
\cline { 5 - 5 } & Success & Degradation & Failure \\
\hline Conduit Penetration Seal & 0.82 & 0.11 & 0.07 \\
\hline Rectangular Penetration Seal & 0.44 & 0.50 & 0.06 \\
\hline Pipe Penetration Seal & 0.79 & 0.18 & 0.03 \\
\hline Floor & 0.89 & 0.11 & 0.00 \\
\hline Wall & 0.94 & 0.06 & 0.00 \\
\hline Building Expansion Joint & 1.00 & 0.00 & 0.00 \\
\hline Door & 1.00 & 0.00 & 0.00 \\
\hline Manhole Seal & 1.00 & 0.00 & 0.00 \\
\hline Duct Bank Seal & 1.00 & 0.00 & 0.00 \\
\hline
\end{tabular}




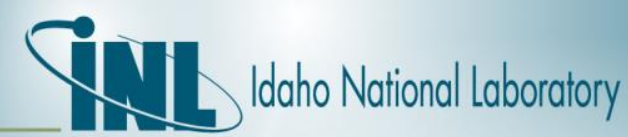

\section{Examples of Previous Flood Barrier Tests}

\section{$>$ Test 1 - Penetration Seals, Ex-Situ}

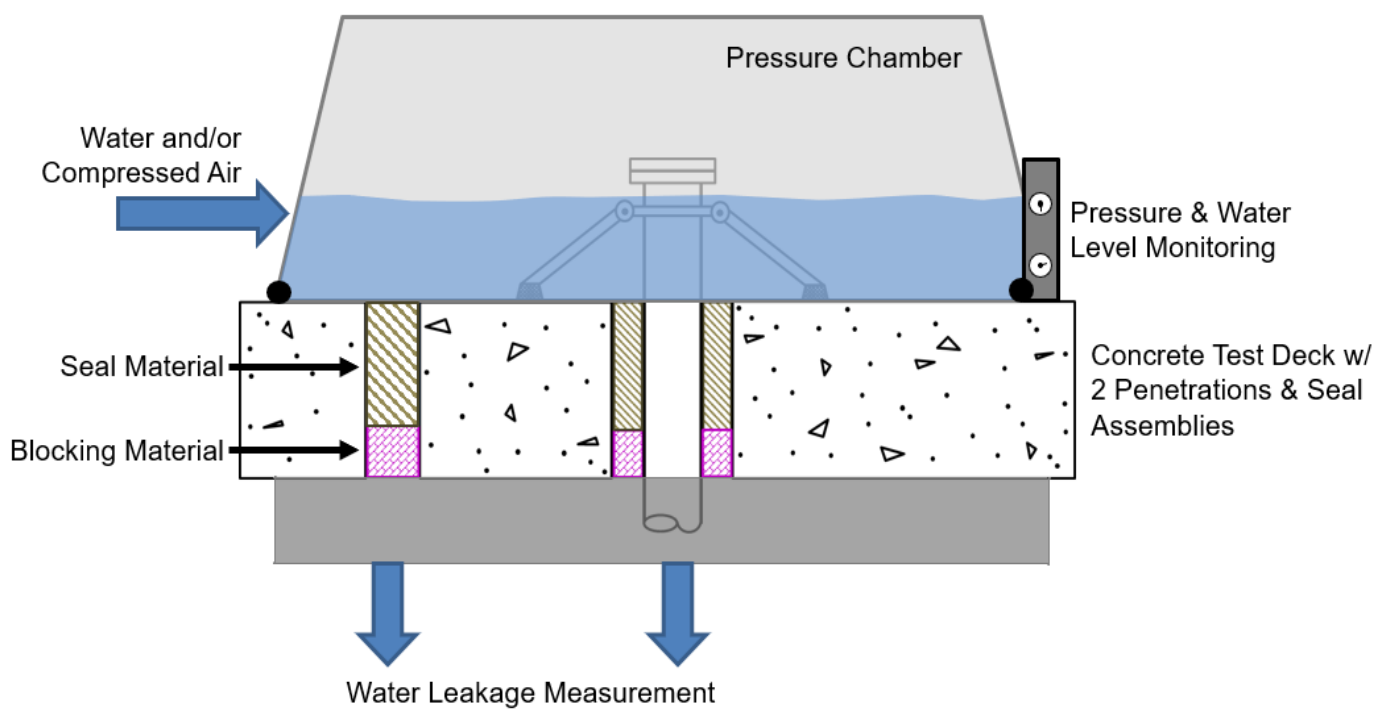

Framatome Laboratory Flood Testing Facility (Lynchburg, VA)

\begin{tabular}{|l|l|}
\hline Flood Barrier Type & Penetration seals \\
\hline Testing Location & Ex-situ \\
\hline Facility Type & $\begin{array}{l}\text { Test deck with pressure } \\
\text { chamber }\end{array}$ \\
\hline Testing Type & $\begin{array}{l}\text { Destructive } \\
\text { Hydrostatic, hydrodynamic }\end{array}$ \\
\hline Included Tests & $\begin{array}{l}\text { Water pressure, duration of } \\
\text { applied pressure, rate of } \\
\text { pressure change }\end{array}$ \\
\hline Test Variables & $\begin{array}{l}\text { Test chamber pressure, water } \\
\text { temperature, water volumetric } \\
\text { flow rates from individual seals }\end{array}$ \\
\hline Test Measuremention & $\begin{array}{l}\text { Until maximum test duration } \\
\text { was exceeded, or seal failure } \\
\text { occurred }\end{array}$ \\
\hline Test Termination & $\begin{array}{l}\text { Test duration, maximum water } \\
\text { pressure, pressure vs. time } \\
\text { graphs, leakage flow rates vs. } \\
\text { time graphs }\end{array}$ \\
\hline Test Outputs & (Numerical)
\end{tabular}




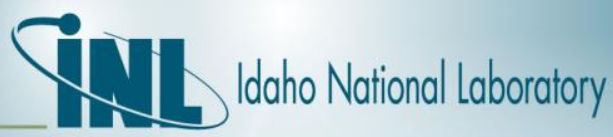

\section{Examples of Previous Flood Barrier Tests (cont.)}

\section{$>$ Test 2 - Non-watertight Doors, Ex-Situ}

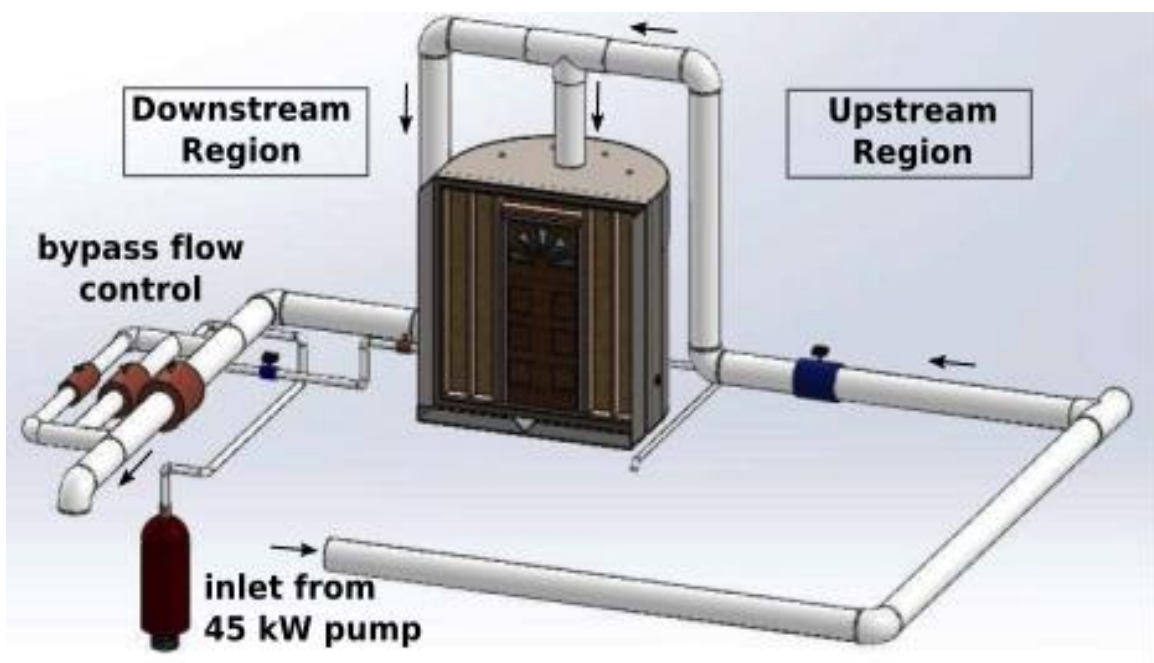

Idaho State University Flood Testing Facility (Pocatello, ID)

\begin{tabular}{|c|c|}
\hline Flood Barrier Type & Doors \\
\hline Testing Location & Ex-situ \\
\hline Facility Type & Tank \\
\hline Testing Type & Destructive \\
\hline Included Tests & Hydrostatic, hydrodynamic \\
\hline Test Variables & Tank filling rate \\
\hline Test Measurements & $\begin{array}{l}\text { Flow rates into the tank, tank } \\
\text { water depth, water } \\
\text { temperature, small leakage } \\
\text { rates, pressures for simulated } \\
\text { hydrostatic head }\end{array}$ \\
\hline Test Termination & $\begin{array}{l}\text { Until door failure, the water } \\
\text { leakage rate equalizing, or } \\
\text { exceeding the filling rate }\end{array}$ \\
\hline $\begin{array}{l}\text { Test Outputs } \\
\text { (Numerical) }\end{array}$ & $\begin{array}{l}\text { Time to failure, failure water } \\
\text { depth, water depth vs. time } \\
\text { graphs }\end{array}$ \\
\hline
\end{tabular}




\section{9}

\section{Examples of Previous Flood Barrier Tests (cont.)}

\section{Tests 3 \& 4 - Temporary Flood Barriers, Ex-Situ}

$\square$ Test Sample

- ACE sandbag barrier

- Three commercial barriers

\begin{tabular}{|c|c|c|}
\hline & Test 3 & Test 4 \\
\hline Flood Barrier Type & Temporary barriers & Temporary barriers \\
\hline Testing Location & Ex-situ & Ex-situ \\
\hline Facility Type & Natural site & Research basin \\
\hline Testing Type & Destructive & Destructive \\
\hline Included Tests & Hydrostatic, hydrodynamic & $\begin{array}{l}\text { Hydrostatic, hydrodynamic, overtopping, debris } \\
\text { impact }\end{array}$ \\
\hline Test Variables & Natural flooding & Water level, wave size, wave duration, debris size \\
\hline Test Measurements & $\begin{array}{l}\text { Water levels in seepage collection pits, time } \\
\text { history of construction/testing/removal of tested } \\
\text { barriers, barrier dimensions }\end{array}$ & $\begin{array}{l}\text { Water levels in seepage collection pits, time } \\
\text { history of construction/testing/removal of tested } \\
\text { barriers, barrier dimensions }\end{array}$ \\
\hline Test Termination & $\begin{array}{l}\text { Until a barrier was overtopped by water flowing } \\
\text { freely over the barrier and exceeding pump } \\
\text { capacity on the protected side }\end{array}$ & $\begin{array}{l}\text { Until maximum test duration was exceeded or } \\
\text { barrier failure occurred }\end{array}$ \\
\hline Test Outputs (Numerical) & $\begin{array}{l}\text { Seepage flow rates, seepage rate vs. wetted } \\
\text { perimeter area graphs, seepage rate vs. stage of } \\
\text { the river graphs, operational concerns (e.g., } \\
\text { ease of construction, barrier durability and } \\
\text { reusability) }\end{array}$ & $\begin{array}{l}\text { Seepage flow rates, barrier displacements, } \\
\text { seepage per linear foot vs. time graphs, seepage } \\
\text { \& overtopping vs. time graphs, operational } \\
\text { concerns (e.g., ease of construction, barrier } \\
\text { durability and reusability) }\end{array}$ \\
\hline
\end{tabular}




\section{Examples of Previous Flood Barrier Tests (cont.)}

\section{$>$ Summary}

$\square$ Tested flood barriers included:

- Permanent barriers

- Temporary barriers

$\square$ All the tests were ex-situ

$\square$ All the tests were destructive

$\square$ All the tested flood barriers were new without aging or degradation 


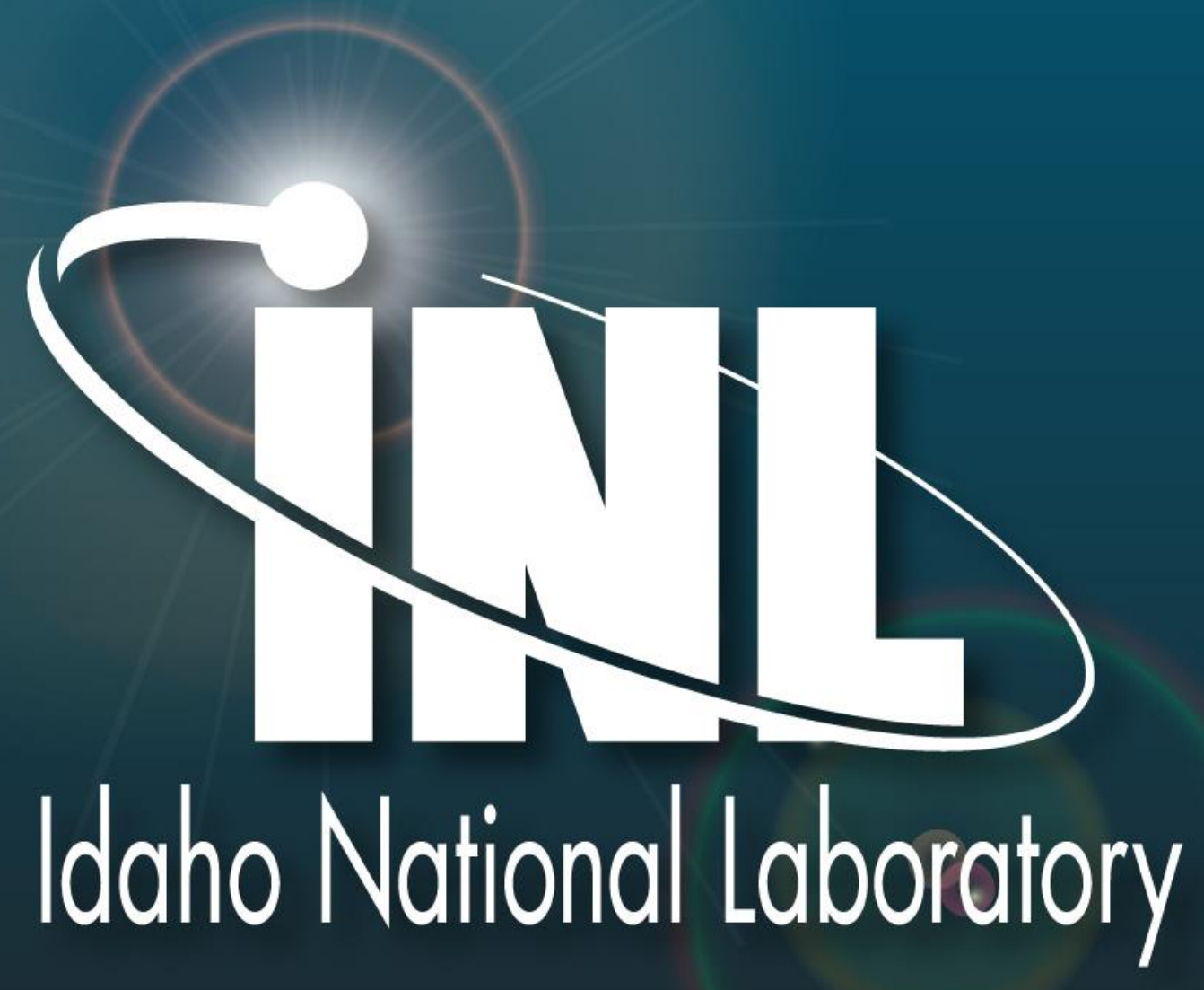

